\title{
Factores socioculturales relacionados con el embarazo reincidente en adolescentes atendidas en el Hospital Docente Madre-Niño San Bartolomé, Lima
}

\author{
Ruth Chura-Muñuico ${ }^{1 *}$, Rosario Campos-Pardo ${ }^{2}$, Lidiana Miranda-Malca ${ }^{2}$ y Susana Asca-Nakamatsu² \\ ${ }^{1}$ E.A.P de Enfermería, Facultad de Ciencias de la Salud, Universidad Peruana Unión, Alt. Km 19; Carretera Central, Chosica, Lima \\ ${ }^{2}$ Hospital Docente Madre Niño San Bartolomé, Av. Alfonso Ugarte 825
}

\begin{abstract}
RESUMEN
Objetivo: Determinar los factores socioculturales relacionados con el embarazo reincidente en adolescentes atendidas en el hospital docente Madre Niño San Bartolomé durante el mes de junio de 2012, Lima. Material y Métodos: Fue una investigación de tipo descriptivo correlacional y corte transversal. La población estuvo conformada por 120 gestantes adolescentes atendidas en consultorios externos, y adolescentes gestantes hospitalizadas en el servicio "Madre adolescente". El instrumento de recolección de datos fue un cuestionario validado por expertos y administrado por las investigadoras. La información fue procesada mediante el paquete estadístico para las ciencias sociales SPSS versión 15 y la significancia estadística de los resultados se estableció mediante la prueba de Chi cuadrado. Resultados: La edad promedio de las adolescentes fue de 17 años; de estado civil solteras en el 51.7\%; el $67.5 \%$ abandonan los estudios; el 34.2\% de las adolescentes residen con sus padres y hermanos. Asimismo, el $50.8 \%$ recibe apoyo económico de su pareja; el $45 \%$ de las adolescentes tiene instrucción secundaria completa; el $85 \%$ respondió que los métodos anticonceptivos sirven para no quedar embarazadas; el $58.3 \%$ usaba el preservativo como método anticonceptivo antes de quedar embarazadas. El 50\% dejó de usar el preservativo por descuido u olvido; el 10.8\% de ellas tuvo embarazo reincidente. Conclusiones: La relación entre los factores socio-culturales y el embarazo reincidente es poco significativa. $(>0.05)$.
\end{abstract}

Palabras clave: Adolescente, embarazo adolescente, embarazo reincidente, factores sociales, factores culturales.

\begin{abstract}
Objective: To determine the socio-cultural factors that relate to repeat pregnancy in adolescents who attended the Mother Child Teaching Hospital San Bartolomé during the month of June 2012, Lima-Peru. Material and Methods: The present research is a descriptive correlational and cross-sectional. The population consisted of 120 pregnant adolescents who were treated in the outpatient and hospitalized pregnant teenagers in service "Teenage Mother". Instrument was used as a survey data collection, the same that was validated by experts and is managed by the researchers in the month of June 2012. The information was processed using the Statistical Package for the Social Sciences SPSS version 15 and statistical significance of the results was established with the chi-square test. Results: The study shows that the average age of the adolescents surveyed is 17 years, $51.7 \%$ were single, also reveals that $67.5 \%$ of adolescents drop out of study. To the question "With whom live at home?", Only $34.2 \%$ of adolescents live with their parents and siblings. On the economic solvency $50.8 \%$ receive financial support from their partner. On cultural factors shows that $45 \%$ of teenagers have completed secondary education. On knowledge of contraceptive methods, $85 \%$ said they used contraception to avoid becoming pregnant. Similarly, $58.3 \%$ of adolescents were using condoms for birth control before becoming pregnant. When asked, "Why did you use?" $50 \%$ said it was because of neglect or oblivion. It also shows that $10.8 \%$ of them have had recurrent pregnancy. Conclusions: The relationship between socio-cultural factors and recurrent pregnancy is insignificant. (>.05).
\end{abstract}

Keywords: Teen, teen pregnancy, repeat pregnancy, social, cultural factors.

*Correspondencia: ruhhis@gmail.com-cel.986655013 


\section{INTRODUCCIÓN}

La OMS (1998) define como adolescencia al "período de la vida en el cual el individuo adquiere la capacidad reproductiva, transitiva de los patrones psicológicos de la niñez a la adultez y consolida la independencia social y económica" que se da entre los 10 y 19 años.

La adolescencia suele ser la edad de las contradicciones e incomprensiones, donde se hace posible el descubrimiento de uno mismo, de los demás y de la ampliación del horizonte individual. El perfeccionamiento y maduración de estas características psicosociales se desplazan, en el adolescente, a edades más avanzadas de la vida, a diferencia de la precoz madurez biológica imperante en esta etapa. Todo esto unido a la falta de información y de educación en el orden sexual, posibilita que las adolescentes se crean aptas para concebir, por lo que se considera este período como importante grupo de riesgo en la salud reproductiva, pues puede dar lugar a una maternidad y una paternidad precoz (Muñoz y col. 1999).

La OMS y la OPS (1998) definen el embarazo adolescente como la concepción en las mujeres menores de 19 años de edad, cuando se considera que termina esta etapa de la vida. El embarazo generalmente es no planificado y, por lo tanto, no deseado.

Según estadísticas mundiales de la OPS (2008), durante el período 2000-2005 el mayor porcentaje de embarazos adolescentes se ubicaron en América Latina y el Caribe (16,3\%) seguido por África $(15,8 \%)$, Norteamérica (12,2\%), y finalmente Europa (7,0\%). En América Latina, el país con mayor incidencia de embarazo adolescente fue Venezuela $(20,5 \%)$, seguido por Brasil (18,0\%), Colombia (16,9\%), Uruguay (15,8\%), Ecuador (14,5\%), Bolivia (14,1\%), Argentina (13,6\%), Paraguay (13,4\%), Perú $(11,6 \%)$ y Chile (10,2\%).

En todo el Perú los embarazos precoces se han incrementado de $12.2 \%$ en el 2006 a $13.7 \%$ en el 2009 . (INEI 2010). Los cuatro departamentos con mayor porcentaje de madres precoces de 12 a 14 años son: Ucayali con 1,2 \%, Madre de Dios con 1,1\%, Loreto y San Martín con $0,9 \%$. Los resultados muestran que son los departamentos de la selva los que poseen el mayor porcentaje de madres adolescentes, siendo los factores determinantes la falta de información sobre métodos anticonceptivos, el estatus económico bajo y el escaso nivel educativo. Lima no es ajena a esta realidad: el 8.1\% son adolescentes, es decir más de 60 mil adolescentes están gestando o son madres (MINSA, 2010).
Dado que el embarazo en la adolescencia es un fenómeno multicausal, pues puede ser una respuesta sintomática a situaciones sociales, así como también una manifestación de las características evolutivas de la adolescente ante determinadas condiciones de vida, es necesario identificar la presencia de importantes factores que predisponen su existencia, entre ellos la misma etapa de la adolescencia, las relaciones intrafamiliares disfuncionales, el poco o nulo conocimiento y práctica de métodos anticonceptivos. Todo esto genera carencias afectivas que no puede resolver la adolescente, siendo el embarazo una forma de escapar de los problemas que se dan en el interior de la familia. (Maddaleno M y col, 2003).

Existen factores que conllevan a este hecho como relaciones sexuales sin protección por falta de educación sexual, el creer que por medio del embarazo se puede retener a la pareja, un escape frente a los problemas familiares, falta de control ante las emociones e impulsos sexuales, actuar bajo la influencia del alcohol o drogas, falta de valores morales y de autoestima, finalmente aquellos que provienen de un incesto o violación, transformándolo aún más en una situación negativa y no deseada. (Pro familia, 2004).

Chirinos (1993) refiere que el embarazo en la adolescencia se incrementa cada vez más y esto se debe a múltiples factores, entre los cuales están la constitución de la familia, las condiciones socioculturales y los medios de comunicación masiva. La familia y las condiciones socioeducativas constituyen un factor esencial, porque generalmente las madres adolescentes provienen de hogares mal constituidos, de madres solteras o divorciadas; de familias sin comunicación adecuada de padre a hija; hogares con un nivel educativo bajo, donde no se da importancia a la educación sexual. El embarazo adolescente ha sido calificado como un problema de salud pública, con un incremento en el número de adolescentes que son madres antes de cumplir los 18 años, y muchas de ellas con embarazos reincidentes.

Por tales motivos, durante la experiencia laboral en la atención de adolescentes, las investigadoras percibieron la necesidad de identificar los factores socioculturales que influyen en la situación descrita. El objetivo principal del estudio fue determinar los factores socioculturales que se relacionan con embarazos reincidentes en adolescentes.

\section{MATERIAL Y MÉTODOS}

El estudio se realizó en el Hospital Nacional Madre Niño San Bartolomé, en Lima (Perú), durante 
el año 2012. La investigación fue de tipo descriptivo correlacional y de corte transversal. La población fue de 120 adolescentes entre las edades de 10 y 19 años captadas en los servicios de consultorios externos y servicio de hospitalización durante el mes de junio del año 2012. Como criterios de inclusión se consideró a adolescentes gestantes comprendidas entre los $10 \mathrm{y}$ 19 años, atendidas en el hospital San Bartolomé y en criterio de exclusión se consideró adolescente gestantes que no deseaban participar en el estudio.

La recolección de datos se realizó a través de un cuestionario autoadministrado, previamente validado por expertos, que estuvo constituido por tres ítemes los cuales fueron: número de gestación actual; factores sociales de las adolescentes (edad, estado civil, ocupación, procedencia, lugar de residencia, inicio de la primera relación sexual, dependencia y apoyo económico y nivel económico) y factores culturales (grado de instrucción, conocimientos sobre los métodos anticonceptivos, uso de métodos anticonceptivos).

Se contó con la autorización de la institución y de las adolescentes gestantes, luego de explicarse el motivo del cuestionario en forma verbal. La información fue procesada con el paquete estadístico para las ciencias sociales SPSS versión 15 y la significancia estadística de los resultados se estableció a través de la prueba de Chi cuadrado.

\section{RESULTADOS}

Tabla 1

Factores sociales que caracterizan a las adolescentes gestantes atendidas en el Hospital Docente Madre-Niño San Bartolomé, Lima, 2012

\begin{tabular}{|c|c|c|}
\hline Factores sociales & $\mathrm{N} .^{\circ}$ & $\%$ \\
\hline \multicolumn{3}{|l|}{ Estado civil } \\
\hline Soltera & 62 & 51.7 \\
\hline Casada & 1 & 0.8 \\
\hline Conviviente & 57 & 47.5 \\
\hline \multicolumn{3}{|l|}{ Ocupación de la adolescente } \\
\hline Estudiante & 33 & 27.5 \\
\hline Su casa & 81 & 67.5 \\
\hline Otros & 6 & 5.0 \\
\hline \multicolumn{3}{|l|}{ Lugar de residencia } \\
\hline Urbano & 85 & 70.8 \\
\hline Rural & 28 & 23.3 \\
\hline Urbano marginal & 7 & 5.8 \\
\hline \multicolumn{3}{|l|}{ La relación con tus padres es } \\
\hline Buena & 88 & 73.3 \\
\hline Regular & 28 & 23.3 \\
\hline Mala & 4 & 3.3 \\
\hline \multicolumn{3}{|c|}{ ¿Hay algún familiar que haya tenido embarazo antes de los 19 años? } \\
\hline Sí & 67 & 55.8 \\
\hline No & 53 & 44.2 \\
\hline \multicolumn{3}{|l|}{ ¿De quién dependes económicamente? } \\
\hline Pareja & 61 & 50.8 \\
\hline Padres & 52 & 43.3 \\
\hline Otros & 7 & 5.8 \\
\hline \multicolumn{3}{|l|}{ Recibe apoyo económico del padre de tu hijo } \\
\hline Sí & 101 & 84.2 \\
\hline No & 19 & 15.8 \\
\hline \multicolumn{3}{|l|}{ ¿En qué nivel económico te consideras? } \\
\hline Alto & 1 & 0.8 \\
\hline Medio & 101 & 84.2 \\
\hline Bajo & 18 & 15.0 \\
\hline Edad del inicio de la primera RS (promedio) & 16 & \\
\hline Edad promedio de la adolescente & 17 & \\
\hline
\end{tabular}


En la tabla 1 se revela que la edad promedio de las adolescentes encuestadas es de 17 años; el $51.7 \%$ de las mismas son solteras; el $67.5 \%$ deja de estudiar dedicándose a las labores domésticas y solo el $27.5 \%$ continúa sus estudios. El $34.2 \%$ de las adolescentes vive sus padres y hermanos, mientras que el $9.2 \%$ vive con otras personas como tíos, abuelos y amigos; la relación de las adolescentes con sus padres es buena en un $73.3 \%$, mientras que el $3.3 \%$ refiere tener una relación mala con ellos. Por otro lado, el $55.8 \%$ de las adolescentes, señala que han tenido un familiar que se embarazó durante la adolescencia; el 50.8\% de ellas recibe apoyo económico de su pareja y el $43.3 \%$ depende de sus padres. El promedio de inicio de las relaciones sexuales fue a los 16 años de edad.

Tabla 2

Factores culturales que caracterizan a las adolescentes gestantes que acuden al Hospital Docente Madre-Niño San Bartolomé, Lima

\begin{tabular}{|c|c|c|}
\hline Factores culturales & $\mathrm{N} .^{\circ}$ & $\%$ \\
\hline \multicolumn{3}{|l|}{ Grado de instrucción de la adolescente } \\
\hline Primaria incompleta & 1 & 0.8 \\
\hline Primaria completa & 6 & 5.0 \\
\hline Secundaria incompleta & 50 & 41.7 \\
\hline Secundaria completa & 54 & 45.0 \\
\hline Superior & 9 & 7.5 \\
\hline \multicolumn{3}{|l|}{ ¿Qué son los métodos anticonceptivos? } \\
\hline Se usa para no quedar embarazada & 102 & 85.0 \\
\hline Evita infecciones de transmisión sexual & 10 & 8.3 \\
\hline No sabe & 8 & 6.7 \\
\hline \multicolumn{3}{|c|}{ ¿Antes de tu embarazo usabas algún método anticonceptivo? } \\
\hline Sí & 70 & 58.3 \\
\hline No & 50 & 41.7 \\
\hline \multicolumn{3}{|l|}{ ¿Qué método usabas? } \\
\hline Píldoras & 14 & 11.7 \\
\hline Inyectables & 6 & 5.0 \\
\hline Preservativo & 48 & 40.0 \\
\hline Otro & 5 & 4.2 \\
\hline No aplica & 47 & 39.2 \\
\hline \multicolumn{3}{|l|}{ ¿Por qué dejaste de usarlos? } \\
\hline Olvido & 60 & 50.0 \\
\hline Otros & 12 & 10.0 \\
\hline No aplica & 48 & 40.0 \\
\hline \multicolumn{3}{|l|}{ ¿Por qué no usabas métodos anticonceptivos? } \\
\hline No conoce & 17 & 14.2 \\
\hline Descuido & 37 & 30.8 \\
\hline Otros & 6 & 5.0 \\
\hline No aplica & 60 & 50.0 \\
\hline
\end{tabular}

En la tabla 2 muestra que el $45 \%$ de las adolescentes tiene secundaria completa, mientras que el $0.8 \%$ tiene instrucción primaria incompleta. En cuanto al conocimiento de los métodos anticonceptivos el $85 \%$ de las adolescentes respondió que los métodos anticonceptivos sirven para no quedar embarazadas.

E1 58.3\% de ellas asegura que usaban algún método anticonceptivo antes de quedar embarazadas, mientras que el $41.7 \%$ no usó método anticonceptivo alguno.

En este caso, refieren no haber usado métodos anticonceptivos por descuido $(30.8 \%)$; las que sí usaban algún método anticonceptivo refieren que no lo hicieron en el momento de la relación sexual por olvido $(50 \%)$. 
Tabla 3

Embarazo reincidente en las adolescentes que acuden al Hospital Docente Madre-Niño San Bartolomé, Lima, 2012

\begin{tabular}{ccc}
\hline Embarazo reincidente & N. ${ }^{\circ}$ & $\%$ \\
\hline No & 107 & 89.2 \\
Sí & 13 & 10.8 \\
\hline Total & 120 & 100.0
\end{tabular}

En la tabla 3 se aprecia que el $89.2 \%$ de las adolescentes no presenta embarazo reincidente,

solo el $10.8 \%$ de estas reincidieron en un segundo embarazo.

Tabla 4

Correlación entre los factores socio culturales con embarazo reincidente según la prueba del Chi cuadrado.

\begin{tabular}{lc}
\hline \multicolumn{1}{c}{ Variable } & Correlación * \\
\hline Factores sociales & 0.46 \\
Edad de la adolescente & 0.62 \\
Edad de la pareja & 0.54 \\
Estado civil de las adolescentes & 0.55 \\
Ocupación de las adolescentes & 0.85 \\
Ocupación de la pareja & 0.19 \\
Procedencia & 0.62 \\
Lugar de residencia & 0.26 \\
Con quién vive en casa & 0.2 \\
Inicio de la primera relación sexual & 0.76 \\
¿Tus padres viven juntos? & 0.48 \\
¿La relación con tus padres es buena? & 0.3 \\
Familiar que se embarazo antes de los 19 años & 0.12 \\
¿De quién depende económicamente? & 0.44 \\
¿Recibes apoyo económico del padre de tu hijo? & 0.94 \\
¿En qué nivel económico te consideras? & \\
Factores culturales & 0.82 \\
Grado de instrucción de la adolescente & 0.07 \\
Grado de instrucción de la pareja & 0.23 \\
¿Qué son los métodos anticonceptivos? & 0.8 \\
¿Antes de tu embarazo usabas algún método anticonceptivo? & 0.87 \\
¿Qué método usabas ante de quedar embarazada? & 0.93 \\
¿Por qué dejaste de usarlos? & 0.27 \\
¿Por qué no usabas algún método? & \\
\hline
\end{tabular}

*Nota: $p<.05$

En la tabla 4 se muestra que todos los factores sociales y culturales evaluados presentan una correlación poco significativa con el embarazo reincidente, es decir $\mathrm{p}>0.05$.

\section{DISCUSIÓN}

En el estudio efectivizado, se encontró que la mitad de las adolescentes tienen padres separados y que la relación con ellos es buena. Estos datos son similares a los de Salaverry y col. (2000) quienes observaron que el $64.4 \%$ de los padres y madres de las adolescentes viven juntos y adoptaron una actitud positiva ante el embarazo de sus hijas. Sin embargo, Alarcón (2009), en un trabajo realizado también en madres adolescentes, encontró que el 52\% tuvo una relación inadecuada con sus padres.

El conocimiento de las adolescentes sobre métodos anticonceptivos y el dejar de usarlos por descuido u olvido, coincide con las investigaciones de Alarcón (2009), quien señala que el $68 \%$ de las adolescentes no usaba ningún método anticonceptivo. Igualmente 
Merino (2011), en su estudio realizado con adolescentes embarazadas, demuestra que la mayoría de ellas no usaba ningún método anticonceptivo a pesar de que los conocían.

El inicio promedio de las relaciones sexuales de las adolescentes ocurre a los 16 años de edad; resultados semejantes a los hallazgos de Merino y Rosales (2011), quienes señalan los 16 años, como edad de inicio de las relaciones sexuales. Gonzales (2003) refiere que la adolescencia y los cambios puberales involucran el inicio de una intensa sexualidad, expresándose a partir de los 14 a 16 años. Estos datos son alarmantes, ya que según la FESAL (2008), 8 de cada 10 mujeres de 15 a 19 años con experiencia sexual, tuvieron al menos un embarazo.

La investigación muestra, del mismo modo, que las adolescentes que quedaron embarazadas dejaron de estudiar, dedicándose a tareas de casa. Pantelides y Binstock (2007) refieren, coincidentemente, que la maternidad en la adolescencia explica el menor nivel de instrucción y que las adolescentes dejan de estudiar por falta de medios económicos e incluso por falta de aceptación de los centros educativos, dedicando el tiempo a su casa y otros asuntos que no favorecen su desarrollo cultural, adicionan que las adolescentes que abandonaron los estudios, raramente vuelven a tener oportunidad de retomarlos.

De las adolescentes encuestadas, la gran mayoría tiene un estado civil de soltera; hallazgo similar con el estudio realizado por Cordero (2003), quien refiere que el $52.3 \%$ de las adolescentes son madres solteras. Por otra parte Chirinos (1993) refiere que el embarazo en adolescentes se incrementa cada vez y la gran mayoría son solteras, fomentando así la disfuncionalidad familiar que, posteriormente, se verá reflejada en el futuro de sus hijos.

En el presente estudio se detectó que la dependencia económica de la mayoría de las adolescentes es afrontada por la pareja. Sin embargo, en su trabajo de investigación Merino y Rosales (2010), encontraron que la dependencia económica de las adolescentes recaía sobre sus padres y un pequeño porcentaje sobre la pareja.

La mayoría de las adolescentes presenta, como antecedente, un familiar que se embarazó antes de los 19 años; información con similitudes al estudio de Salaverry (2003), quien considera que la familia disfuncional, familia en crisis, madre o hermana con embarazo, influyen en el embarazo adolescente.

Asimismo, el presente trabajo muestra que hay una correlación poco significativa entre el embarazo reincidente y los factores sociales y culturales, sin embargo, Paukku (2003) muestra que el embarazo reincidente se asocia a uno de los factores culturales como el mal uso de los métodos anticonceptivos y sociales como la ausencia de la figura paterna, mala relación madre-hija y ausencia de apoyo familiar para la anticoncepción que llevan a la falta de motivación para evitar un nuevo embarazo.

\section{CONCLUSIONES}

Los factores sociales que caracterizan a las gestantes adolescentes son los siguientes: la edad promedio es de 17 años, abandono de sus estudios y continuidad de convivencia con los padres, reciben apoyo económico en baja proporción de su pareja, la relación con sus padres es buena, la mayoría de ellas tiene un familiar cercano que se embarazó durante la adolescencia y son de nivel económico medio.

Entre los factores culturales que caracterizan a las gestantes adolescentes figuran: grado de instrucción secundaria, tienen conocimientos acerca de los métodos anticonceptivos pero no los practican, y no usan método anticonceptivo durante el acto sexual por olvido o descuido.

Además, la mayoría de las adolescentes embarazadas no reinciden en el embarazo. Se encontró relación poco significativa ente los factores sociales y culturales con el embarazo reincidente. 


\section{REFERENCIAS BIBLIOGRÁFICAS}

Alarcón R. (junio, 2009) Factores que influyen en el embarazo en la adolescencia. Revista Cubana de Enfermería vol. 25, 1-2 Ciudad de la Habana. Cuba.

Chirinos, M (octubre, 1993). Embarazo en adolescencia. Rev. Cubana de Enfermería. Recuperado de http://. bvs.sld.cu/revistas/san/vol5_1_01/san06101.pdf.

Cordero, A. (junio, 2003). Factores sociales que influyen en las adolescentes embarazadas. Unidad de Gineco Obstetricia. Hospital Universitario “Antonia María Pineda". Barquisimeto. Venezuela.

Encuesta Nacional de Salud Familiar FESAL (2008) Informe Final. Asociación demográfica Salvadoreña. El Salvador

Gonzales, G. (2003). Factores psicosociales que se asocian a la reincidencia del embarazo en adolescente chilenas. Revista de la Sociedad Chilena de Obstetricia y Ginecología Infantil y de la Adolescencia.; 10: 71-72.

Instituto Nacional de Estadística e Informática INEI (2010). Perú: situación social de las madres adolescentes, 2007.

Maddaleno M, (2003). Salud y desarrollo de adolescentes y Jóvenes en Latinoamérica y el Caribe: desafios para la próxima década. Salud Pública supl. 1:S132-S139. México.

Merino E. \& Rosales E. (junio, 2011). Factores que influyen en el embarazo de las adolescentes inscritas en el programa de atención materna de unidad de salud altavista, El Salvador.

Ministerio de Salud e Instituto Materno Perinatal
MINSA/ IMAPE. (2010). Curso Nacional de Adolescencia y salud Reproductiva. Perú.

Muñoz E.(octubre, 1999). Anticoncepción y sexualidad en la adolescencia. Departamento de Obstetricia y Ginecología. Hospital Universitario. Volumen 58, 2. España.

Organización Panamericana de la Salud (2008). El embarazo en la adolescencia precoz. Rev. Panamericana de la Salud Vol. 3, 4 Estados Unidos.

Organización Mundial de la Salud/ Organización Panamericana de la Salud (OMS/OPS). (1998). Fondo de Población de las Naciones Unidas. Embarazo en adolescencia: Lineamientos para el desarrollo de investigaciones y aspecto socioculturales. Estados Unidos.

Pantelides, Ey Binstock, G. (julio, 2007). La fecundidad adolescente en la Argentina al comienzo del siglo XXI. Rev. Argent. Social. v 5, 9 Buenos Aires. Argentina.

Paukku (2003). Adolescents Contraceptive Use and Pregnancy History: Is there a Pattern. American College of Obstetricians and gynecologist. $101: 534-538$.

Asociación para la promoción y defensa de la familia (2004) Educación sexual en los hijos y escuelas, Profamilia. Buenos Aires Argentina. Disponible en: http://www.profamilia.org.ar/publicaciones.html.

Salaverry y Col. (noviembre, 2003). Embarazo en adolescentes de Lima. Perú. Actualidad Psicológica. Disponible en Http://www.angelfire. com/pe/actualidadpsi/embarazo.html. 